\title{
The effect of sports VR training for improving human body composition
}

\author{
Hyo Taek Lee and Yoon Sang Kim ${ }^{*}$
}

\begin{abstract}
The purpose of this study is to find out the effect of sports virtual training with applied sports science and information and communication technology (ICT) for adults. We have developed a 4-week workout program that focuses on improving basic endurance, strength, and function. The experiment is to derive the kinematic data for ergonomic evaluation of indoor sports device combined with a virtual image and to compare and evaluate the pre- and post-body composition. Twenty-one participants were selected, and each training program was conducted considering individual differences and levels. Results showed that 4-week sports virtual reality (VR) training program developed in this study turned out to be appropriate as a sport VR training one to improve body composition and health. Although it is difficult to generalize the effect to all human, it is meaningful to study some ways of reducing the heterogeneity and improving the training efficiency in the situation where the sports VR system is gradually being applied to the exercise.
\end{abstract}

Keywords: Sports VR training, Body composition, Sports biomechanics

\section{Introduction}

VR (virtual reality) technology provides immersion and fun to operators and a positive effect of increasing learning effectiveness in the field of education and training [1, 2]. It should be noted that the end user of VR and AR (augmented reality) is human. In other words, efforts to make end users as easy, comfortable, mentally and physically burdens as possible should be studied as VR and AR pursue users' needs (fun, immersion, etc.). For example, a system that poses a threat to safety, as infants and toddlers who have seen Pokémon videos in the 1990s, which caused headaches, vomiting, and seizures in Japan, are not likely to be socially acceptable. Recently, there have been actively developed products that incorporate images in indoor fitness equipment (bicycles, running machines, etc.) [3]. In many cases, 2D, 3D, or HMD (head mounted display)-based VRs are applied to existing mechanical fitness device to increase exercise immersion and eliminate boredom. In this case, due to the image fatigue caused by the VR image, a physical imbalance may be induced after the end of the exercise. Smaller products are more likely to experience image fatigue because they give a stereoscopic effect on a smaller screen than a regular monitor, and image fatigue remains in the body for a certain period of time even after VR stimulation is removed [4]. Most of the related studies have used quantitative image qualities such as flicker fusion frequency tester (EEG) to measure the degree of image fatigue depending on the types of image stimuli (2D, 3D, HMD-based VR). Also, research is also being conducted on how visual fatigue, one of image fatigue that is generated by image, is affected by personality. However, research on the effect of VR-applied sports device on physical exercise ones other than mental influences has not been actively conducted yet. Physical education classes using ICT are becoming an alternative to overcome weak physical education facilities and environments. ICT education methods such as internet, various software, and simulation program utilization can provide opportunities for various experiences by overcoming the limit of time and space. Therefore, the purpose of this study is to investigate the effect of sports VR training (4-week aerobic/anaerobic exercise) for body composition, which thus provides theoretically the exercise program for effective health care.

\footnotetext{
* Correspondence: yoonsang@koreatech.ac.kr

BioComputing Lab, Institute for Bio-engineering Application Technology, Departent of Computer Science and Engineering, KOREATECH, Cheonan, Republic of Korea
} 


\section{Methods}

\subsection{Subjects}

Twenty-three male university students who had experience using VR game participate in this study. But those in the experimental group who did not participate in the exercise sessions more than three times during the total period of 4 weeks were excluded from the results analysis, which resulted in there ultimately being 21 male subjects $(23.71 \pm 1.87$ years $, \quad 175.81 \pm 4.12 \mathrm{~cm}, \quad 67.23 \pm 14.55 \mathrm{~kg}$, $22.96 \mathrm{~kg} / \mathrm{m}^{2}$ ) in the experimental group. Exclusion criteria had a history of neuromuscular diseases and related operations within the preceding 6 months and limited range of motion. All subjects participated in the experiments after being sufficiently informed of the contents of the experiments. To ensure postural accuracy and safety, each subject was given sufficient time to practice. Size of the subjects was calculated by using G*POWER 3.1 version program [5], and the number of calculated sample was 19 participants with 0.05 significance level, 0.95 power, with 0.8 large effect size and therefore, it satisfied the number of participants (Table 1).

\subsection{Sports VR system}

A sports VR system used for the experiment was fixed on a flat surface. The task was executed on the sports device, which consists of a platform on wheels that moves left and right, parallel metal rails. Rubber belts fasten the platform to the rails and ensured that it regains its resting position in the middle of the apparatus [6, 7]. Participants should pass many of obstacles (based on participant's level) in VR environment about $1 \sim 3 \mathrm{~min}$ as shown in Fig. 1. The sports VR system that passes between the obstacles accelerates when sliding with the shortest distance, and it is a way to determine the winner by deriving the accuracy of the turn and the time record. We conducted the experiment with medium levels of elasticity strength according to subject's body weight, condition, and levels.

The sports VR system consists of the distance measurement using the IR sensor and the reflector, and the actual body movement, muscle strength, balance, and resistance band. Participations use their lunch time and the after-school activity time for 12 trials ( 3 days $\times 30 \mathrm{~min} \times 4$ weeks). In addition to the sports, it is composed of contents to be able to learn the actual skill of the sports before the start of the game. The warm-up exercises were performed for approximately $5 \mathrm{~min}$, the main exercises were done for approximately 20-30 min depending on the exercises performed, and the wrap-up exercises were done for approximately $5 \mathrm{~min}$. The 4 -week period was divided into

Table 1 Subject characteristics

\begin{tabular}{lllll}
\hline$N$ & Age & Height & Weight & BMI \\
\hline 21 & $23.71 \pm 1.87$ & $175.81 \pm 4.12$ & $67.23 \pm 14.55$ & 22.96 \\
\hline
\end{tabular}

the adaptive period (week 1), the development period (weeks 2-3), and the maintenance period (week 4) as shown in Fig. 1a, which were performed in a same sequence order to avoid compromising the results.

The intensity of the sports VR training was controlled at level 11 on the rating of perceived exertion (RPE) scale and at a percentage of maximal heart rate (\%HR max) of $80 \%$. Body and balance measurements before and after applying the sports VR training program were performed with a body composition analyzer (Inbody 520, Korea) [8]. The body composition test measured the ratio and amount of constituting the human body to the body mass index $(r)$. BMI is a measure of obesity based, divided by body weight $(\mathrm{kg})$ divided by the square of your height (cm) [9]. BMI can be used in a variety of fields because it has the usefulness to measure individual obesity worldwide. The body composition analyzer can assess not only weight, BMI, body fat, and muscle mass but also obesity in the abdominal region and muscle development according to body part. For the body composition measurements, the subjects held electrode handles in both hands in a vertical position with their socks off and maintained the correct posture for approximately $1 \mathrm{~min}$.

Descriptive statistics were used for the physical characteristics of the groups and the measurement data. A paired-sample $t$ test was conducted to examine the difference between before and after the sports VR training program, and post hoc analysis was used to examine the correlation between variables. The statistical significance level for the measured values was set at $p<0.05$, and the data were statistically processed using IBM SPSS Statistics version 20.0 .

\section{Results and discussion}

The purpose of this study is to investigate the effects of sports VR training. The VR training (program) was designed to actively reflect the movement of the body. In addition, we tried to induce their participation continuously. In this way, students are interested in the program that they have not easily experienced before and have been exercising steadily. In this way, students were interested in the program that they have not easily experienced before. As a result (Table 2, Fig. 2), the body water and protein showed significantly $(p<.05)$ higher after 4 week of sports VR training $(36.13 \mathrm{~kg})(9.74 \mathrm{~kg})$ than pre training $(35.72 \mathrm{~kg})$ $(9.60 \mathrm{~kg})$. As a result of the body fat pre- and post-exercise, there were $18.50 \mathrm{~kg}$ (pre), $17.73 \mathrm{~kg}$ (post), and skeletal muscle mass was $26.98 \mathrm{~kg}$ (pre) and $27.36 \mathrm{~kg}$ (post) with significant difference $(p<.05)$. As body fat decreased, skeletal muscle mass increased; thus, there seems to be a correlation between body fat and skeletal muscle mass. Fat mass and body fat (\%) also decreased in post-exercise with significant difference $(p<.05)$, and both arm and leg also increased post-exercise as well. 


\section{a}

\begin{tabular}{|c|c|c|c|}
\hline $\begin{array}{l}\text { Workout 1week. } \\
\text { 3days }\end{array}$ & $\begin{array}{l}\text { SoundBody - PROSKI } \\
\text { Basic endurance, coordingation, balance and strength }\end{array}$ & & \\
\hline WARM-UP & $\begin{array}{l}\text { Warm Up } 5 \mathrm{~min} \\
\text { stretching on the floor } \\
\text { check ROM and intensity }(1-6 / 1-10)\end{array}$ & $\begin{array}{l}\text { REP/TIME } \\
\text { 5MIN }\end{array}$ & $\begin{array}{l}\text { REST / INT } \\
\text { FREE }\end{array}$ \\
\hline TECHINQUE & $\begin{array}{l}\text { Basci Skill 10min } \\
\text { ver.1 (tutorial) } \\
\text { ver.2 (competiton) }\end{array}$ & $3 \operatorname{set} \times 1.5 \mathrm{~min}$ & $1.5 \mathrm{~min} / \mathrm{LOW}^{*}$ \\
\hline CARDIO/STRENGHT & $\begin{array}{l}\text { Functional Training 15min } \\
\text { ver.3 (real ski 3) }\end{array}$ & 3 set $\times 3.5 \mathrm{~min}$ & $1.5 \mathrm{~min} / \mathrm{LOW}^{*}$ \\
\hline COOL DOWN & $\begin{array}{l}\text { Cool down } 5 \mathrm{~min} \\
\text { stretching on the floor }\end{array}$ & $5 \mathrm{MIN}$ & FREE \\
\hline
\end{tabular}

\begin{tabular}{|clcc|}
\hline $\begin{array}{c}\text { Workout 2week. } \\
\text { 3days }\end{array}$ & $\begin{array}{l}\text { SoundBody } \\
\text { asic endurance, coordingation, balance and strength }\end{array}$ & & \\
\hline WARM-UP & $\begin{array}{l}\text { WARM UP 5min } \\
\text { stretching on the floor } \\
\text { check ROM and intensity(1-6/1-10) }\end{array}$ & $\begin{array}{c}\text { REP/TIME } \\
5 \text { MIN }\end{array}$ & $\begin{array}{c}\text { REST / LOW INT. } \\
\text { FREE }\end{array}$ \\
\hline TECHINQUE & $\begin{array}{l}\text { Basci Skill 10min } \\
\text { A-2 Workout : 1(2)-2(2)-3(2)-1(2) }\end{array}$ & 4 set $\times 2$ min & 0.5 min/Medium \\
\hline \hline CARDIO/STRENGHT & $\begin{array}{l}\text { Functional Training 15min } \\
\text { A-3 Workout : 1(2)-2(2)-3-4-5-6 }\end{array}$ & 5 set $\times 2$ min & 1 min/Medium \\
\hline \hline COOL DOWN & $\begin{array}{l}\text { Cool down } 5 \text { min } \\
\text { stretching on the floor }\end{array}$ & 5 MIN & FREE \\
\hline
\end{tabular}

\section{b}

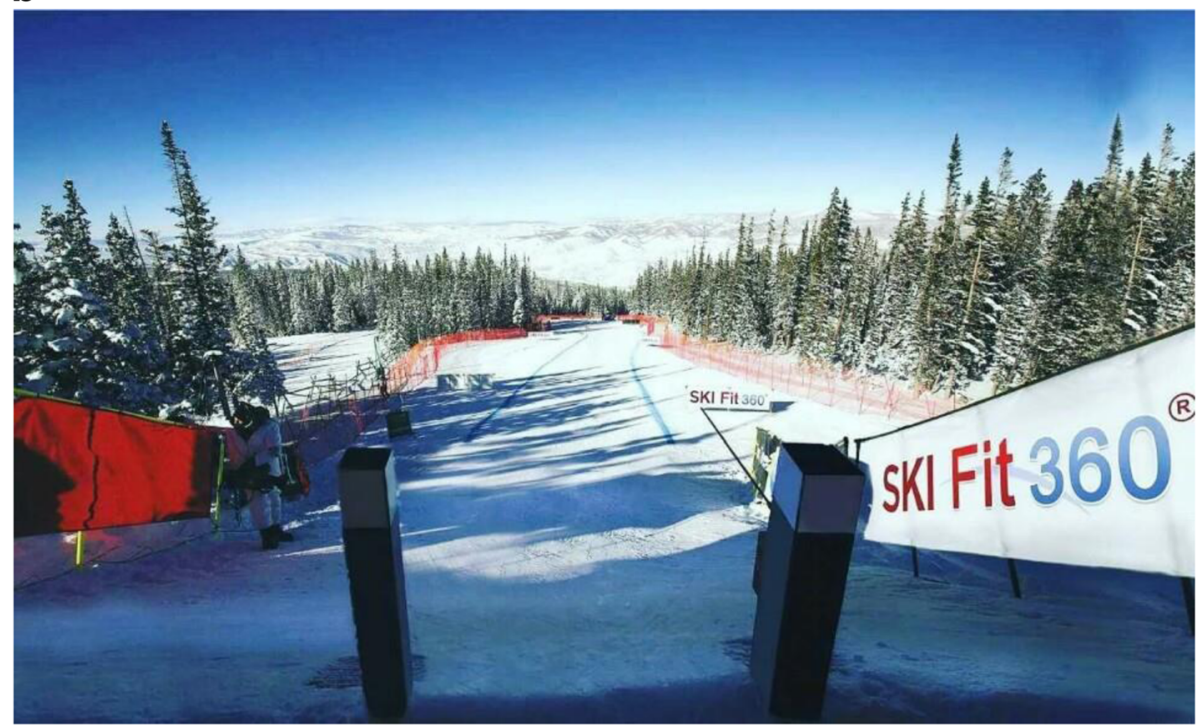

Fig. 1 The proposed sports VR system. a 4-week workout. b A snapshot of virtual training images integrated with platform

Therefore, the body fat, muscle strength, and balance (ratio) between upper and lower body were improved in the sports VR training program. Thus, we can expect that the longer the training period, the greater the range of motion of the body joint angle can be interpreted as maximizing the effect of exercise. It also seems that carrying out the exercise while watching the VR screen (images) showed more exercise amount and concentration (participation rate) than ordinary exercise. American College of Sports Medicine (ACSM) underscored the importance of physical strength with regard to health over physical strength in terms of motor skills based on the fact that the decline in four capacities of body composition, cardiorespiratory endurance, muscular strength, and muscular endurance and flexibility significantly increases the probability of diagnoses of lifestyle diseases and health impairments. One of the most critical elements of physical fitness is cardiorespiratory endurance. Resistance exercises enhance muscle mass and stimulate muscle activities, thus efficiently aligning the body parts and maintaining good posture. They also improve one's ability to perform various exercises requiring muscle strength, endurance, and energy; thus, they can positively influence basic health functions such as metabolism and cardiovascular functioning $[10,11]$. However, resistance exercises, an inadequate exercise carries a risk of injury even when the exercises are well timed and well equipped [12]. This study attempted to examine how sports VR training can 
Table 2 The effect of sports VR training on human body composition

\begin{tabular}{lllll}
\hline & Pre-exercise & Post-exercise & $T$ & $p$ \\
\hline Body water ( $\ell$ ) & $35.72 \pm 7.49$ & $36.13 \pm 7.66$ & -2.748 & $.012^{*}$ \\
Protein (kg) & $9.60 \pm 2.03$ & $9.74 \pm 2.09$ & -2.687 & $.014^{*}$ \\
Minerals (kg) & $3.41 \pm 0.67$ & $3.43 \pm 0.70$ & -.795 & .436 \\
Body fat (kg) & $18.50 \pm 7.52$ & $17.73 \pm 6.91$ & 2.263 & $.035^{*}$ \\
Body weight (kg) & $67.23 \pm 14.55$ & $67.02 \pm 14.50$ & .558 & .583 \\
Skeletal muscle mass (kg) & $26.98 \pm 6.14$ & $27.36 \pm 6.32$ & -2.539 & $.020^{*}$ \\
Fat mass (kg) & $18.50 \pm 7.52$ & $17.73 \pm 6.91$ & 2.263 & $.035^{*}$ \\
BMl (kg/m $\left.{ }^{2}\right)$ & $22.96 \pm 4.00$ & $22.87 \pm 3.97$ & .709 & .487 \\
Body fat (\%) & $27.13 \pm 7.85$ & $26.15 \pm 7.37$ & 2.842 & $.010^{*}$ \\
Abdominal fat (0-1) & $0.87 \pm 0.07$ & $0.87 \pm 0.06$ & .301 & .766 \\
R arm (kg) & $2.47 \pm 0.77$ & $2.52 \pm 0.78$ & -3.159 & $.005^{* *}$ \\
Ratio (\%) & $86.72 \pm 9.43$ & $88.61 \pm 9.43$ & -3.546 & $.002^{* *}$ \\
L arm (kg) & $2.44 \pm 0.75$ & $2.49 \pm 0.77$ & -3.447 & $.003^{* *}$ \\
Ratio (\%) & $85.51 \pm 8.76$ & $87.49 \pm 9.09$ & -4.061 & $.001^{* *}$ \\
Trunk (kg) & $21.35 \pm 4.58$ & $21.14 \pm 4.67$ & .445 & .661 \\
Ratio (\%) & $92.56 \pm 5.49$ & $93.87 \pm 5.42$ & -3.880 & $.001^{* *}$ \\
R leg (kg) & $7.98 \pm 1.80$ & $8.01 \pm 1.81$ & -.834 & .414 \\
Ratio (\%) & $99.28 \pm 10.68$ & $99.88 \pm 10.69$ & -1.533 & .141 \\
L leg (kg) & $7.93 \pm 1.77$ & $7.98 \pm 1.78$ & -1.357 & .190 \\
Ratio (\%) & $98.71 \pm 10.57$ & $99.54 \pm 10.52$ & -2.065 & .052 \\
\hline *p<.05, **p<.01 & & & &
\end{tabular}

improve the postural alignment and body composition of an adult. The sports VR training can help to use minor muscles that are not frequently used in daily activities, without implementing running or large movements of the body. It is an exercise that can enhance body function and muscle strength, while addressing incorrect posture, fatigue, muscle pain, low back pain, shoulder and neck pain, and stress [13]. The sports VR training, which was created by ski concept, is a form of exercise that relaxes body tension and strengthens deep muscles by using the body weight of the participant. This kind of program is known to be useful in enhancing lower limb function in various targets. This study confirms that sports VR training has a positive impact on body postural alignment and composition in adults, which can be attributed to the fact that sports VR training is performed symmetrically and strengthens the core muscles. Moreover, the results showed that muscle mass, amount of body fat, and balance are critical in maintaining trunk postural alignment. Because the actions of the muscles should come first in order to maintain the skeletal form and the postural alignment.

\section{Conclusions}

We investigated the effects of 4-week sports VR training which is a type of VR game-based program. The sports VR training system provides a combination of horizontal, sagittal, and coronal plane motion moving transversely. For the athletes, they are movements that need to improve their skills and prevent injury $[6,10]$. Whereas, it is an exercise that can improve the function and strength of the body while exercising the small muscles that are not normally used by the general people and can eliminate the wrong posture, fatigue, pain, and stress. In many previous studies [11-15], simulator-based sports exercise has also been shown to increase the stability of the trunk by influencing bilateral movements. In this study, we also confirmed that the alignment of the body coordination and the muscle mass increases, and we found that body fat decreased and muscle mass increased through the 4-week training. Therefore, appropriate sports VR training (game-based) is seen as an important factor in the trunk alignment, and it also affects the strength, balance, and quality of life of the lower extremities. Recently, studies

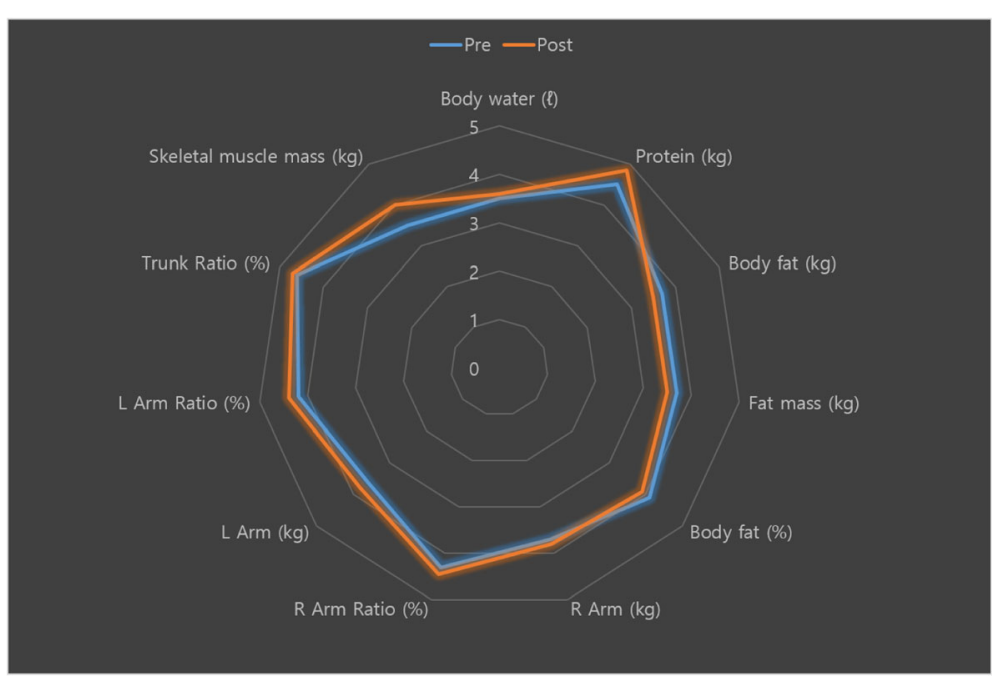

Fig. 2 The effect of sports VR training on human body composition (5-point scale) 
with various age groups and materials included VR gears [14, 16-18] have been continuing and have been taking place as an effective training method. In this study, sports VR training which may be helpful for aerobic exercise and may strengthen cardiopulmonary showed better effect on coordination (body composition). Changes in body composition during sports VR training exercise may contribute to the stability of posture as well as the balance needed for daily life. Based on this research, it has been confirmed that even a 4-week exercise can positively affect body composition. And it is expected that it can be applied to the training (program) to improve human body composition with various target including rehab and elderly, and to help to find ways to develop immersion and actual exercise effect during stimulating people's interests.

\section{Abbreviations}

BMl: Body mass index; HMD: Head mounted display; RPE: Rating of perceived exertion; VR: Virtual reality

\section{Acknowledgements}

The authors thank the editor and anonymous reviewers for their helpful comments and valuable suggestions.

\section{Funding}

Not applicable.

\section{Availability of data and materials}

The datasets used and/or analyzed during the current study are available from the corresponding author on reasonable request.

\section{About the authors}

Hyo Taek Lee He obtained B.S., M.S., and Ph.D. degrees in Sports Biomechanics from Pukyong National University, Busan, Korea, in 2007, 2010, 2013, respectively. Currently, he is a CEO at the BioHealthCore CO., Ltd., Cheonann, Korea. His research areas include Sports VR training and Sports biomechanics. Yoon Sang Kim He obtained B.S., M.S., and Ph.D. degrees in Electrical Engineering from Sungkyunkwan University, Seoul, Korea, in 1993, 1995, 1999, respectively. Currently, he is a full professor at the School of Computer and Science Engineering, Korea University of Technology Education (KOREATECH), Cheonan, Korea. His research areas include Virtual simulation, Power-IT technology, and Bio-informatics.

\section{Authors' contributions}

LHT analyzed the participant data regarding kinematic characteristics. KYS was a major contributor in writing the manuscript. All authors read and approved the final manuscript.

\section{Competing interests}

The authors declare that they have no competing interests.

\section{Publisher's Note}

Springer Nature remains neutral with regard to jurisdictional claims in published maps and institutional affiliations.

Received: 21 August 2018 Accepted: 28 November 2018

Published online: 19 December 2018

\section{References}

1. D.J. Kim, D.H. Koo, Development and application of elementary science IT convergence education program. The Journal of Korea Elementary Education 24(3), 119-138 (2013)

2. S.J. Lee, Y.S. Kim, Effects on physiological factors of intelligent health VR exercise protocol is suitable for chronic stroke patients using the Mobile app. Korean Society for The Study of Physical Education 21(2), 97-114 (2016). https://doi.org/10.15831/JKSSPE.2016.21.2.97
3. S.J. Han, S.U. Kim, J.H. Cho, K.C. Koo, Ergonomic evaluation of indoor bike coordinated with virtual images. Journal of Digital Convergence 15(5), 443451 (2017)

4. S.J. Han, S.U. Kim, K.C. Koo, K.J. Lee, M.S. Cho, Effects of virtual reality images on body stability: Focused on hand stability. Journal of Digital Convergence 15(8), 391-400 (2017)

5. F. Faul, E. Endfelder, A. Buchner, A.G. Lang, Statistical power analyses using G*power 3.1: Tests for correlation and regression analyses. Behav Res Methods 41(4), 1149-1160 (2009). https://doi.org/10.3758/BRM.41.4.1149

6. H.T. Lee, Y.J. Kim, KI.M. YS, Kinematic study with and without ski boots using ski simulator. Science \& Sports 32, 9-14 (2017)

7. H.T. Lee, H.O. Oh, H.S. Han, K.Y. Jin, H.L. Roh, Effect of mat Pilates exercise on postural alignment and body composition of middle-aged women. J Phys Ther Sci 28, 1691-1695 (2016)

8. Body composition analyzer (Inbody 520, Republic of Korea). https://www. inbody.com. Accessed 21 August 2018

9. J.K. Byeun, Y.B. Park, The effect of Nintendo Wii boxing program on healthrelated physical fitness in students with intellectual disabilities. Korean Journal of Adapted Physical Activity 23(4), 181-191 (2015). https://doi.org/ 10.17006/kjapa.2015.23.4.181

10. P.A. Fausto, M. Giuseppe, P. Nicola, Comparative evaluation of two skiing simulators as functional training devices for recreational skiers. Journal of Sports Science \& Medicine 12, 151-158 (2013)

11. K. Nakazato, P. Scheiber, E. Muller, Comparison between the force application point determined by portable force plate system and the center of pressure determined by pressure insole system during alpine skiing. Sports Engineering 16(4), 297-307 (2013). https://doi.org/10.1007/s12283-013-0119-x

12. K. Hirose, H. Doki, A. Kondo, Dynamic analysis and motion measurement of ski turns using inertial and force sensors. Procedia Engineering 60, 355-360 (2013). https://doi.org/10.1016/j.proeng.2013.07.082

13. H.T. Lee, H.L. Roh, Y.S. Kim, Kinematic characteristics of the lower extremity during a simulated skiing exercise in healthy participants. J Phys Ther Sci 28 626-631 (2016). https://doi.org/10.1589/jpts.28.626

14. A. Ancillao, E. Palermo, S. Rossi, Validation of ankle strength measurements by means of a hand-held dynamometer in adult healthy subjects. Journal of Sensors 11 (2017)

15. H.T. Lee, H.L. Roh, Y.S. Kim, Cardiorespiratory endurance evaluation using heart rate analysis during ski simulator exercise and the Harvard step test in elementary school students. J Phys Ther Sci 28, 641-645 (2016). https://doi. org/10.1589/jpts.28.641

16. S. Cloix, G. Bologna, V. Weiss, T. Pun, D. Hasler, Low-power depth-based descending stair detection for smart assistive devices. EURASIP Journal on Image and Video Processing 2016, 33 (2016)

17. D. Naranjo-Hernandez, L.M. Roa, J. Reina-Tosina, G. Barbarov-Rostan, O Galdamez-Cruz, Smart device for the determination of heart rate variability in real time. Journal of Sensors (3):1-11 (2017). https://doi.org/10.1155/2017/ 8910470

18. Y. Kong, S. Lee, J. Lee, Y. Nam, A head-mounted goggle-type videooculography system for vestibular function testing. EURASIP Journal on Image and Video Processing 218, 28 (2018)

\section{Submit your manuscript to a SpringerOpen ${ }^{\circ}$ journal and benefit from:}

- Convenient online submission

- Rigorous peer review

- Open access: articles freely available online

High visibility within the field

- Retaining the copyright to your article

Submit your next manuscript at $\boldsymbol{\nabla}$ springeropen.com 